\title{
Estadificación del cáncer de recto mediante ultrasonografía endoscópica: correlación con la estadificación histológica
}

\author{
J. J. Vila, F. J. Jiménez, R. Irisarri, A. Martínez, E. Amorena y F. Borda \\ Servicio de Aparato Digestivo. Hospital de Navarra. Pamplona
}

\section{RESUMEN}

Objetivo: nuestro objetivo fue evaluar la precisión diagnóstica (PD) en nuestro medio de la ecoendoscopia (USE) para la estadificación del cáncer de recto (CR).

Material y métodos: incluimos de manera prospectiva a todos los pacientes con CR estadificados en nuestra unidad entre septiembre de 2002 y febrero de 2006 en una base de datos. Seleccionamos aquellos pacientes en los que se había realizado una estadificación completa con USE(UTN) y fueron intervenidos quirúrgicamente sin tratamiento neoadyuvante. Consideramos la estadificación histológica (pTN) como patrón oro, y comparamos los resultados de la uTN previa con los de la pTN. Calculamos la sensibilidad (S), especificidad (E), valores predictivos positivo (VPP) y negativo (VPN) y PD para cada estadio $T$, y estadio $N$ considerado como positivo o negativo. Calculamos la concordancia entre la uTN y la pTN utilizando el índice de kappa para el estadio N, y el mismo índice con ponderación cuadrática para el estadio $\mathrm{T}$.

Resultados: ciento veinte pacientes con $\mathrm{CR}$ fueron estadificados en nuestra unidad, cumpliendo 36 criterios de inclusión y fueron analizados en este estudio ( 21 hombres, 15 mujeres). La edad media fue de 68,53 $\pm 10,15$ años (rango $=48-90$ ). La PD de la uT global y uN fueron del 83 y $72 \%$ respectivamente. Obtuvimos una S, E, VPP, VPN y PD de 91, 100, 100, 96 y 97\% para el T1; 82, 88, 75, 91 y 86\% para el T2; 86, 91, 86, 91 y 89\% para el T3; y 14, 86, 20, 80 y $72 \%$ para el estadio N respectivamente. El índice de kappa para la estadificación $\mathrm{T}$ fue de 0,87 (concordancia "muy buena" entre uT y pT); y de 0,005 para la estadificación N (concordancia "pobre").

Conclusiones: en nuestra experiencia, la PD de la uTN del cáncer de recto alcanza el 83 y el $72 \%$ respectivamente, resultados acordes con lo disponible en la literatura. La uT del cáncer de recto muestra una concordancia "muy buena" con la $\mathrm{pT}$.

Palabras clave: Ecoendoscopia. Cáncer de recto. Estadificación tumoral. Tratamiento neoadyuvante.

\begin{abstract}
Objective: our aim was to evaluate the accuracy of endosonography (EUS) in our experience, to stage rectal cancer.

Material and methods: we prospectively included all patients with rectal cancer staged in our unit from September 2002 until February 2006 in a database. We selected those patients who had a complete EUS examination and were surgically treated without neoadjuvant therapy. Once we had the results of the histopathological staging (pTN), which was considered the gold standard, we compared the results of the previous EUS staging (uTN) with those of the pTN. We calculated the sensitivity, specificity, positive predictive value, negative predictive value and accuracy for each $\mathrm{T}$ stage, and for $\mathrm{N}$ staging considered as $\mathrm{N}$ positive or negative. We also calculated the global accuracy for $\mathrm{T}$ stage. We also calculated the agreement of uTN with pTN staging using the kappa index for $\mathrm{N}$ stage, and quadratic weighted kappa index for T stage
\end{abstract}

Results: we staged 120 patients with rectal cancer during the mentioned period. Of these, 36 patients met inclusion criteria and were evaluated, 21 women and 15 men. Mean age was 68,53 \pm 10,15 yo (range: $48-90$ ). Global T stage accuracy was $83 \%$. N stage accuracy was $72 \%$. We obtained a S, E, PPV, NPV and A of $91,100,100,96$ and $97 \%$ for T1; $82,88,75,91$ and $86 \%$ for T2; 86, 91, 86, 91 and 89\% for T3; and 14, 86, 20, 80 and $72 \%$ for $\mathrm{N}$ stage respectively. Kappa value for $\mathrm{T}$ stage was 0,87 indicating a "very good" agreement between uT and pT according to the kappa index criteria. Kappa value for $\mathrm{N}$ stage agreement was 0,005 ; "poor" according to the same criteria.

Conclusions: in our experience, the diagnostic accuracy of EUS for $\mathrm{T}$ and $\mathrm{N}$ staging of rectal cancer is $83 \%$ and $72 \%$ respectively, similar results as previously published. uT staging for rectal cancer shows a "very good" agreement with pT staging.

Key words: Endosonography. Rectal neoplasms. Neoplasm staging. Neoadjuvant therapy.

Vila JJ, Jiménez FJ, Irisarri R, Martínez A, Amorena E, Borda F. Estadificación del cáncer de recto mediante ultrasonografía endoscópica: correlación con la estadificación histológica. Rev Esp Enferm Dig 2007; 99: 132-137.

Recibido: 18-07-06.

Aceptado: 01-12-06.

Correspondencia: Juan J. Vila Costas. Servicio de Aparato Digestivo. Hospital de Navarra. C/ Irunlarrea, 3. 31008 Pamplona. e-mail: xoanxose@hotmail.com 


\section{INTRODUCCIÓN}

El cáncer colorrectal representa un importante problema de salud en los países occidentales con una incidencia en ascenso en las últimas décadas. En Navarra el cáncer colorrectal es el tercero más frecuente en hombres y el segundo en mujeres. En el periodo comprendido entre 1998 y 2000 se diagnosticaron en nuestra comunidad 398 nuevos casos de cáncer de recto, con una tasa ajustada a la población mundial anual de 17 casos/100.000 habitantes para hombres y 8,3 casos/100.000 habitantes para mujeres (1).

El cáncer de recto además presenta un alto índice de recurrencia local tras la cirugía, que varía entre el 30\% y el $65 \%$ en diferentes estudios (2). Se ha demostrado que aquellos pacientes con cáncer rectal localmente avanzado (estadios T3-4Nx o TxN1-2) presentan un menor índice de recidiva tumoral y una mayor supervivencia cuando se les administra tratamiento oncológico neoadyuvante preoperatorio (3-5).

Es vital por tanto realizar una precisa estadificación preoperatoria del cáncer de recto, siendo hoy en día la ultrasonografía endoscópica (USE) una de las exploraciones que ofrece una mejor precisión diagnóstica con este fin. La precisión diagnóstica de la USE para la estadificación T varía entre el 69 y el 97\% (6), mientras que para la estadificación $\mathrm{N}$ ronda el 70-75\% (7).

Como suele ser habitual, los primeros estudios publicados a propósito de una técnica de nueva aparición suelen ofrecer resultados excelentes $(8,9)$, mientras que la generalización en el uso de esta técnica y una moderación del entusiasmo inicial llevan a unos resultados más variables, y a menudo menos impresionantes en cuanto a su precisión diagnóstica (10). En este sentido y en lo concerniente al papel de la USE en el cáncer de recto, podemos ver en la literatura que existe una relación inversa entre la precisión diagnóstica obtenida y el tamaño muestral de los diferentes estudios. Además, las precisiones diagnósticas más altas las encontramos en los estudios más antiguos, en posible relación con el fenómeno previamente comentado. Una posible explicación para estos hechos es que exista un sesgo de publicación de forma que se tienda a publicar aquellos estudios con los mejores resultados, aunque el tamaño muestral sea pequeño (7). De esta forma, cabría pensar que la capacidad real de la USE para realizar una correcta estadificación del cáncer rectal sea inferior a la publicada en la literatura.

Ante esta situación, nos planteamos el objetivo de analizar la precisión diagnóstica de la estadificación del cáncer de recto mediante la USE en nuestro medio, tomando como referencia la estadificación histológica.

\section{MATERIAL Y MÉTODOS}

Para hacer una valoración de la USE para la estadificación del cáncer de recto, incluimos de forma prospectiva a todos aquellos pacientes con diagnóstico de novo de cáncer de recto estadificados en nuestra unidad desde septiembre de 2002 hasta febrero de 2006. Como criterios de inclusión en el presente estudio definimos: tumores que afectaran a los últimos $15 \mathrm{~cm}$ de colon (11), en los que disponíamos de un estudio ecoendoscópico completo, y que fueron intervenidos quirúrgicamente sin tratamiento oncológico neoadyuvante previo, y por tanto, aquellos pacientes en los que disponíamos de una estadificación histológica completa no artefactada por el tratamiento neoadyuvante. Esta estadificación histológica fue la que tomamos como patrón oro para hacer una valoración de la estadificación previa mediante USE.

Para hacer una valoración de la precisión diagnóstica de la USE en función de la localización del tumor, dividimos el recto en tres tercios: tercio superior desde 12 hasta $15 \mathrm{~cm}$, tercio medio desde 8 hasta $12 \mathrm{~cm}$ y tercio inferior por debajo de $7 \mathrm{~cm}$.

Como exploración ecoendoscópica rectal completa entendemos aquella en la que se hace un estudio ecográfico al menos desde la bifurcación de los vasos ilíacos. Como preparación para la USE rectal administramos dos enemas de limpieza de $250 \mathrm{cc}$ en las horas previas a la exploración, con una separación entre ambos enemas de aproximadamente 12 horas. Todas las USE fueron realizadas sin sedación y sin tratamiento analgésico, por un único endoscopista y con un ecoendoscopio radial mecánico (Olympus, GF UMQ130) (Olympus Europa, Hamburgo). Para realizar la estadificación utilizamos la clasificación TNM (AJCC Cancer Staging Manual, Sixth edition, Springer Verlag, New York Inc.; 2002).

En nuestro hospital existe un Comité de Oncología Digestiva en el que se valoran los resultados de la estadificación de estos pacientes, aplicando un protocolo aceptado por los servicios de Oncología, Radiología, Aparato Digestivo y Cirugía General, según el cual a aquellos pacientes con estadificación preoperatoria de $\mathrm{T} \geq 3 \mathrm{y} / \mathrm{o} \mathrm{N}$ positivo se les administra tratamiento neoadyuvante con radioterapia a una dosis de 45 Gy y quimioterapia concomitante basada en el 5-FU.

Para realizar el estudio comparativo además de los estadísticos descriptivos, utilizamos el índice de correlación de kappa con ponderación cuadrática para la comparación del estadio T, y el índice de kappa no ponderado para el estadio N. Este índice nos da un valor dentro de una escala donde 1 representa la máxima concordancia, y 0 representa la concordancia debido al azar (Tabla I). Además del valor del índice de kappa, aportamos el valor de $\mathrm{p}$ (considerado significativo por debajo de 0,05$)$ para dicho índice y el intervalo de confianza (IC) para un nivel de confianza del 95\%. Calculamos además la sensibilidad, especificidad, valores predictivos positivo y negativo, y precisión diagnóstica de la USE para la estadificación T global y N, para cada estadio $\mathrm{T}$ y también estos valores según la localización del tumor. El análisis estadístico lo realizamos con el programa SPSS (versión 12.0) (SPSS, Chicago, 1L, USA). 
Tabla I. Escala del índice de kappa

\begin{tabular}{cc}
\hline Valor de kappa & Fuerza de la concordancia \\
\hline$<0,20$ & Pobre \\
$0,21-0,40$ & Débil \\
$0,41-0,60$ & Moderada \\
$0,61-0,80$ & Buena \\
$0,81-1$ & Muy buena \\
\hline
\end{tabular}

\section{RESULTADOS}

Durante el periodo señalado estadificamos en nuestra unidad de endoscopia a 120 pacientes con cáncer de recto. De estos, 36 cumplían criterios de inclusión en el presente estudio y fueron analizados. Se trataba de 21 hombres $(58,3 \%)$ y 15 mujeres $(41,7 \%)$. La edad media fue de $68,53 \pm 10,15$ años, con rango: 48-90 años. En cuanto a la localización de los tumores, en 14 pacientes el tumor se encontraba en tercio inferior; en 7 pacientes a nivel de tercio medio y en 15 pacientes en tercio superior. La USE estadificó el cáncer como T1 en 10 pacientes $(27,8 \%)$, como T2 en 12 pacientes (33,3\%), y como T3 en 14 pacientes $(38,9 \%)$. Por otro lado, en 5 pacientes $(13,9 \%)$ la USE determinó la existencia de adenopatías sospechosas de malignidad. En el estudio histológico finalmente 11 pacientes $(30,6 \%)$ eran $\mathrm{T} 1 ; 11$ pacientes $(30,6 \%)$ eran $\mathrm{T} 2$, y 14 pacientes $(38,9 \%)$ eran T3. En el estudio histológico se demostró la presencia de metástasis linfáticas en 7 pacientes $(19,4 \%)$. Los datos referentes a la comparación entre la estadificación mediante USE y estadificación histológica los mostramos en las tablas II y III. En la estadificación $\mathrm{T}$ sobreestadificamos a 3 pacientes $(8,3 \%)$, dos pacientes que identificamos mediante la USE como T3 y realmente eran $\mathrm{T} 2$, y un paciente que clasificamos ecoendoscópicamente como T2 y en el estudio histológico resultó ser un T1. Por otro lado, infraestadificamos también a 3 pacientes $(8,3 \%)$, todos ellos T3 en el estudio histológico que fueron identificados en la ecoendoscopia como $\mathrm{T} 2$.

Según estos resultados obtuvimos unos valores de sensibilidad, especificidad, valores predictivos positivo y negativo, y precisión diagnóstica que mostramos en la tabla IV. La precisión diagnóstica obtenida para la estadificación T global mediante USE fue del $83 \%$. Al tener en cuenta la localización del tumor, obtuvimos una precisión diagnóstica para el estadio $\mathrm{T}$ a nivel de tercio superior del $73,3 \%$; en tercio medio del $100 \%$, y en tercio inferior del $85,7 \%$.

Calculamos la correlación entre la estadificación TN mediante USE y la estadificación histológica utilizando el índice de kappa con ponderación cuadrática para la estadificación $\mathrm{T}$ y no ponderado para la estadificación $\mathrm{N}$. Así, obtuvimos un valor del índice kappa de 0,87 (IC =

Tabla II. Comparación de la estadificación T mediante USE y estadificación histológica

\begin{tabular}{lccccc}
\hline \multicolumn{7}{c}{ Estadificación histológica } \\
\hline Estadificación USE & \multicolumn{7}{c}{$T 2$} & $T 3$ & Total \\
& T1 & $10(27,8 \%)$ & 0 & 0 & $10(27,8 \%)$ \\
& T2 & $1(2,7 \%)$ & $8(22,2 \%)$ & $3(8,3 \%)$ & $12(33,3 \%)$ \\
& T3 & 0 & $2(5,5 \%)$ & $12(33,3 \%)$ & $14(38,8 \%)$ \\
& Total & $11(30,5 \%)$ & $10(27,8 \%)$ & $15(41,7 \%)$ & $36(100 \%)$ \\
\hline
\end{tabular}

Tabla III. Comparación de la estadificación N mediante USE y estadificación histológica

\begin{tabular}{lcccc}
\hline & \multicolumn{3}{c}{ Estadificación histológica } \\
\hline Estadificación USE & N positivo & N negativo & Total \\
& N positivo & $1(2,7 \%)$ & $4(11,1 \%)$ & $5(13,8 \%)$ \\
& N negativo & $6(16,6 \%)$ & $25(69,4 \%)$ & $31(86,1 \%)$ \\
& Total & $7(19,4 \%)$ & $29(80,5 \%)$ & $36(100 \%)$ \\
\hline
\end{tabular}

Tabla IV. Resultados de sensibilidad (S), especificidad (E), valor predictivo positivo (VPP), valor predictivo negativo (VPN) y precisión diagnóstica (PD) de la estadificación mediante USE para cada estadio T y estadio N

\begin{tabular}{|c|c|c|c|c|c|c|}
\hline & $n$ & $S$ & $E$ & $P P V$ & $N P V$ & $P D$ \\
\hline T1 & 11 & $91 \%$ & $100 \%$ & $100 \%$ & $96 \%$ & $97 \%$ \\
\hline $\mathrm{T} 2$ & 11 & $82 \%$ & $88 \%$ & $75 \%$ & $91 \%$ & $86 \%$ \\
\hline T3 & 14 & $86 \%$ & $91 \%$ & $86 \%$ & $91 \%$ & $89 \%$ \\
\hline $\mathrm{N}$ & 36 & $14 \%$ & $86 \%$ & $20 \%$ & $80 \%$ & $72 \%$ \\
\hline
\end{tabular}


0,56-0,93; $\mathrm{p}<0,05)$ para la estadificación T, correspondiente a una correlación muy buena según esta clasificación. El valor del índice de kappa para la estadificación $\mathrm{N}$ fue de 0,005 (IC $=-0,34-0,35 ; p=0,24$ ) lo que corresponde a una correlación pobre.

\section{DISCUSIÓN}

La USE es una de las técnicas que ofrece una mayor precisión diagnóstica para la estadificación del cáncer de recto, siendo esta una de las principales indicaciones de esta exploración (12). Se han publicado estudios comparativos en los que la USE ha demostrado su superioridad para la estadificación $\mathrm{T}$ del cáncer de recto con respecto al TAC (2), y valores superiores o similares a la RNM. Bien es cierto que la RNM ha experimentado una importante mejoría en sus resultados con los avances tecnológicos incorporados. La RNM de alta resolución mejora los resultados obtenidos con RNM con bobina rectal o corporal (6), constituyendo hoy en día una buena alternativa a la USE (13). El problema de la RNM es su alto coste, disponibilidad limitada y sus discretos resultados en la estadificación de tumores en estadio precoz cuando se compara con la USE $(13,14)$. A cambio, permite un estudio completo de todo el mesorrecto, es menos operador dependiente que la USE, e identifica la afectación de la fascia mesorrectal y por tanto puede determinar la indemnidad del margen circunferencial de resección, lo cual puede ser determinante como comentaremos más adelante (15).

La USE tampoco está exenta de dificultades, sus resultados pueden estar influenciados por diversos factores como contacto inadecuado entre el transductor y el tumor debido a la presencia de aire, heces o una superficie tumoral irregular; errores de interpretación, con una tendencia a la sobrestadificación; defectos anatómicos por biopsias previas o polipectomía, inflamación peritumoral que puede favorecer la sobrestadificación, y una exploración ecográfica incompleta (16). Cuando alguna de estas situaciones se produce, es posible que los resultados de la USE no sean del todo fiables y debemos plantearnos el repetir la exploración cuando dicho factor de confusión haya desaparecido, o realizar una estadificación mediante RNM (15).

Además de mediante USE radial, podemos realizar una estadificación ecográfica de los tumores rectales mediante otras técnicas como la ecografía endorrectal con sonda rígida, USE sectorial y minisondas ecográficas. Con la primera se han descrito resultados ligeramente superiores a los de USE radial, pero su utilización queda prácticamente limitada a los tumores situados en los dos tercios inferiores de recto y tumores no estenosantes $(17,18)$. Además, no permite valorar la presencia de adenopatías a nivel de la bifurcación iliaca en la mayoría de los pacientes, por lo que nosotros preferimos realizar la estadificación con USE radial. También se han utilizado las minisondas ecográficas para la estadificación del cáncer de recto, ofreciendo muy buenos resultados para diferenciar los tumores limitados a la mucosa de los que invaden la submucosa, lo cual puede ser muy útil en aquellos pacientes en los que se plantee una resección endoscópica o transanal del tumor (19). Por último, también se ha utilizado la USE sectorial en la estadificación del cáncer de recto con la gran ventaja de que permite realizar la punción de adenopatías perirrectales o iliacas, mejorando de esta manera la estadificación $\mathrm{N}$. Esto en algún estudio se asoció a una mayor supervivencia al realizar una estadificación más precisa y seleccionar mejor a los pacientes (20).

En los últimos años, y como ya comentamos en la introducción de este artículo, la utilidad de la USE para realizar la estadificación de cáncer de recto ha sido discutida debido a la publicación de varios estudios en los que se obtuvieron peores resultados que los publicados previamente $(21,22)$. Estos estudios fueron realizados con un tamaño muestral importante, pero en ellos no se incluyen los tumores localmente avanzados en los que la ecoendoscopia ha demostrado una mayor precisión diagnóstica $(23,24)$. Esto podría representar un sesgo de selección que empeorara los resultados. Por otro lado, los propios autores reconocen que los resultados son explorador dependiente.

Ante estos nuevos datos, ha sido publicado recientemente un artículo de lectura muy recomendable, en el que se aprecia una relación inversa entre el tamaño muestral y la precisión diagnóstica obtenida para la estadificación del cáncer rectal mediante USE. Además, en este artículo también se señala que aquellos trabajos en los que se obtienen mejores resultados son los más antiguos. Los autores proponen como posible explicación la existencia de un sesgo de publicación según el cual tienden a publicarse aquellos trabajos en los que se obtienen mejores resultados, creándose unas expectativas con respecto a la estadificación del cáncer de recto mediante USE que pudieran no ser reales (7). En nuestro caso, obtuvimos unos resultados similares a los obtenidos por otros autores en estudios con tamaño muestral igual al nuestro $(25,26)$. Este tamaño muestral no es muy importante y nuestro trabajo podría entrar a formar parte de ese grupo de trabajos que empeoran el sesgo de publicación. En nuestra defensa hemos de decir que nuestro objetivo era conocer la utilidad y fiabilidad de la USE para la estadificación del cáncer de recto en nuestro medio en las condiciones que trabajamos habitualmente, y estos resultados son el reflejo de la situación real de nuestro hospital a lo largo de los últimos dos años y medio.

Llama la atención que pacientes estadificados en nuestra serie con la USE como T3 o N positivo hayan sido intervenidos quirúrgicamente sin tratamiento neoadyuvante previo. Como ya hemos comentado, en nuestro hospital se ha constituido un Comité de Oncología Digestiva en el que se aplica un protocolo según el cual a los tumores estadificados como $\mathrm{T} 3 \mathrm{o}$ superior y/o $\mathrm{N}$ positivo, se les ad- 
ministra tratamiento oncológico neoadyuvante (3-5). Sin embargo, las decisiones terapéuticas son individualizadas y en ocasiones pacientes en estadio localmente avanzado son intervenidos quirúrgicamente sin tratamiento neoadyuvante, bien porque se trate de un paciente de edad avanzada, bien porque la localización del tumor lo facilita, o bien porque el propio paciente rechace el tratamiento neoadyuvante. Esta situación se describe también en otros trabajos $(2,27)$. En nuestro estudio puede constituir un sesgo de selección, al incluir tan sólo a un determinado grupo de pacientes con estadio localmente avanzado. Aceptando que esto es posible, valoramos también que la precisión diagnóstica obtenida en los tumores T3 es similar a los de estadio más precoz, por lo que consideramos que esta precisión diagnóstica puede estar cercana a la realidad. Por otro lado, este posible sesgo de selección podría tener cierta influencia en la mala correlación obtenida en la estadificación $\mathrm{N}$, al no estar incluidos la mayoría de los tumores estatificados como $\mathrm{N}$ positivo. Esto no tendría tanta influencia sobre la correlación del estadio T dado que en este análisis existen tres categorías y dos de ellas (T1 y T2) sí incluyen a todos sus pacientes, y por tanto la influencia del sesgo de selección de la tercera categoría tendría menor peso en el análisis. La correlación de la estadificación mediante USE con la estadificación histológica para el estadio T fue muy buena.

Los resultados obtenidos en nuestros pacientes son similares a los publicados en series más cortas por otros grupos nacionales, aunque el diseño de nuestro estudio cambia con respecto a estos trabajos previos. Así, en nuestro caso analizamos sólo los resultados en tumores con estadio más precoz (T1/2-N0, y algún T3 con determinadas características ya comentadas), mientras que en un trabajo de Novell y cols. (28) incluyen todos los tumores debido a que este estudio fue publicado antes de que se confirmara la bondad del tratamiento oncológico neoadyuvante; y en otro trabajo más reciente de García Sanchez y cols. (17), por un lado distinguen un primer grupo similar al nuestro compuesto por 17 pacientes, y por otro lado analizan la respuesta al tratamiento neoadyuvante en los pacientes con estadio superior. Esto último es criticable en el sentido de que dan por hecho que la estadificación ecoendoscópica era correcta en todos estos pacientes, lo cual es falso, y esto puede llevar a error a la hora de evaluar la respuesta al tratamiento neoadyuvante.

Sin embargo, el sistema de estadificación TN está siendo actualmente criticado fundamentalmente porque no discrimina entre un tumor T3 limitado, con un amplio margen de resección circunferencial, de un tumor T3 avanzado en el que el margen de resección es pequeño o incluso está afectado $(6,29)$. Se ha demostrado que la distancia del tumor al plano de resección mesorrectal circunferencial es el predictor más potente de recurrencia local, y no el estadio T (6,27,30-32). Es importante por tanto que las exploraciones de imagen utilizadas en la estadificación identifiquen la fascia mesorrectal y puedan medir la distancia entre el tumor y esta (29). Una distan- cia del tumor a la fascia mesorrectal menor de $5 \mathrm{~mm}$ se considera inadecuada para una buena resección quirúrgica (33). En este sentido, el problema de la USE es que no siempre consigue visualizar correctamente la fascia mesorrectal. La RNM sí ha demostrado su utilidad, pudiendo predecir el margen de resección circunferencial con un $97 \%$ de confianza cuando la distancia es de al menos $6 \mathrm{~mm}$, con una buena concordancia interobservador (33).

Como conclusión podemos decir que según nuestros datos la USE ofrece una alta precisión diagnóstica para la estadificación TN del cáncer de recto, con una muy buena correlación con la estadificación histológica para el estadio T. Sin embargo, y en espera de lo que nos puedan ofrecer los últimos avances de la tecnología como la ecoendoscopia con contraste o la ecoendoscopia 3D, puede que esta alta precisión en la estadificación TN pierda peso en el futuro ante la importancia creciente que se le está dando a la distancia del tumor a la fascia mesorrectal como predictiva de un margen de resección circunferencial adecuado. Esta distancia que puede ser medida de forma fiable con la RNM, es difícilmente identificable con la USE. Ante esta situación es posible que según todo lo comentado, en el futuro debamos realizar una USE para una estadificación inicial y en aquellos tumores estadificados como T3 ampliar el estudio mediante una RNM para hacer una valoración del margen de resección circunferencial.

\section{BIBLIOGRAFÍA}

1. Ardanaz E, Moreno C, Pérez de Rada Arístegui ME, Ezponda C, Navaridas N. Incidencia de cáncer en Navarra (1998-2000). An Sist Sanit Navar 2004; 27: 373-80.

2. Harewood GC, Wiersema MJ, Nelson H, Maccarty RL, Olson JE, Clain JE, et al. A prospective, blinded assessment of the impact of preoperative staging on the management of rectal cancer. Gastroenterology 2002; 123: 24-32.

3. NIH Consensus conference. Adjuvant therapy for patients with colon and rectal cancer. JAMA 1990; 264: 1444-50.

4. Improved survival with preoperative radiotherapy in resectable rectal cancer. Swedish rectal cancer trial. N Engl J Med 1997; 336: 980-7.

5. Kapiteijn E, Marijnen CA, Nagtegaal ID, Putter H, Steup WH, Wiggers $\mathrm{T}$, et al. Preoperative radiotherapy combined with total mesorectal excision for resectable rectal cancer. N Engl J Med 2001; 345 : 638-46.

6. Beets-Tan RG, Beets GL. Rectal cancer: How accurate can imaging predict the $\mathrm{T}$ stage and the circumferential resection margin? Int $\mathrm{J}$ Colorectal Dis 2003; 18: 385-91.

7. Harewood GC. Assessment of publication bias in the reporting of EUS performance in staging rectal cancer. Am J Gastroenterol 2005; 100: 808-16.

8. Saitoh N, Okui K, Sarashina H, Suzuki M, et al. Evaluation of echographic diagnosis of rectal cancer using intrarectal ultrasonic examination. Dis Colon Rectum 1986; 29: 234-42.

9. Pappalardo G, Reggio D, Frattaroli FM, et al. The value of endoluminal ultrasonography and computed tomography in the staging of rectal cancer: a preliminary study. J Surg Oncol 1990; 43: 219-22.

10. Meining A, Dittler HJ, Wolf A, et al. You get what you expect? A critical appraisal of imaging methodology in endosonographic cancer staging. Gut 2002; 50: 599-603.

11. Nelson H, Petrelli N, Carlin A, Couture J, Fleshman J, Guillem J, et 
al. Guidelines 2000 for colon and rectal cancer surgery. J Natl Cancer Inst 2001; 93: 583-96.

12. Fusaroli P, Caletti G. Endoscopic ultrasonography: current clinical role. Eur J Gastroenterol Hepatol 2005; 17: 293-301.

13. Bartram C, Brown G. Endorectal ultrasound and magnetic resonance imaging in rectal cancer staging. Gastroenterol Clin North Am 2002; 31: 827-39.

14. Bipat S, Glas AS, Slors FJ, Zwinderman AH, Bossuyt PM, Stoker J. Rectal cancer, local staging and assessment of lymph node involvement with endoluminal US, CT, and MR imaging- a meta-analysis. Radiology 2004; 232: 773-83.

15. Zammit M, Jenkins JT, Urie A, O’Dwyer PJ, Mohillo RG. A technically difficult endorectal ultrasound is more likely to be inaccurate. Colorectal Dis 2005; 7: 486-91.

16. Kim JC, Yu CS, Jung HY, Kim HC, et al. Source of errors in the evaluation of early rectal cancer by endoluminal ultrasonography. Dis Colon Rectum 2001; 44: 1302-9.

17. García Sanchez MV, Reyes López A, López Vallejos P, Mata Tapia I, Jiménez Sánchez JR. Valor de la ecografía endorrectal en el cáncer de recto y su implicación en el tratamiento radioquimioterápico previo a la cirugía. Rev Esp Ecogr Dig 2004; 6: 5-10.

18. Varas MJ, Marco C. Ecoendoscopia y cáncer de recto. Rev Esp Enferm Dig 1999; 91: 611-3.

19. Abad Belando R, Varas Lorenzo MJ. ¿Las minisondas ecográficas transendoscópicas son una alternativa a la USE? Rev Esp Ecogr Dig 2004; 6: 46-51.

20. Harewood GC. Assesment of clinical impact of endoscopic ultrasound on rectal cancer. Am J Gastroenterol 2004; 99: 623-7.

21. Marusch F, Koch A, Schmidt U, Zippel R, Kuhn R, Wolff S, et al. Routine use of transrectal ultrasound in rectal carcinoma: Results of a prospective multicenter study. Endoscopy 2002; 34: 385-90.

22. García-Aguilar J, Pollack J, Lee SH, Hernández de Anda E, Mellgren A, Wong WD, et al. Accuracy of endorectal ultrasonography in preoperative staging of rectal tumors. Dis Colon Rectum 2002; 45: $10-5$.
23. Tio TL, Coene PP, van Delden OM, Tytgat GN. Colorectal carcinoma: Preoperative TNM classification with endosonography. Radiology $1991 ; 179: 165-70$.

24. Shimizu S, Tada M, Hawai K. Use of endoscopic ultrasonography for the diagnosis of colorectal tumors. Endoscopy 1990; 22: 31-4.

25. Sentovich SM, Blatchford GJ, Falk PM, et al. Transrectal ultrasound of rectal tumors. Am J Surg 1993; 166: 638-42.

26. Thaler W, Watzka S, Martin F, et al. Preoperative staging of rectal cancer by endoluminal ultrasound vs magnetic resonance imaging. Preliminary results of a prospective, comparative study. Dis Colon Rectum 1994; 37: 1189-93.

27. Lee SH, Hernandez de Anda E, Finne CO, Madoff RD, GarcíaAguilar J. The effect of circumferential tumor location in clinical outcomes of rectal cancer patients treated with total mesorectal excision. Dis Colon Rectum 2005; 48: 2249-57.

28. Novell F, Cáceres JP, Novell-Costa F, Piulachs J, Targarona EM, Trias M. La ecografía endorrectal en la evaluación del estadiaje de las neoplasias de recto. Cir Esp 1992; 52: 247-51.

29. Reerink O, Mulder NH, Szabo BG, Sluiter WJ, Wiggers T, Bongaerts $\mathrm{AHH}$, et al. Developments in treatment of primary irresectable rectal cancer. Colorectal Dis 2004; 6: 406-17.

30. Wibe A, Rendedal PR, Svensson E, Norstein J, Eide TJ, Myrvold $\mathrm{HE}$, et al. Prognostic significance of the circumferential resection margin following total mesorectal excision for rectal cancer. $\mathrm{Br} \mathrm{J}$ Surg 2002; 89: 327-34.

31. Nagtegaal ID, Marijnen CA, Kranenbarg EK, van de Velde CJ, van Krieken JH. Circumferential margin involvement is still an important predictor of local recurrence in rectal carcinoma: not one millimeter but two millimeters is the limit. Am J Surg Pathol 2002; 26: 350-7.

32. Brown G, Daniels IR. Preoperative staging of rectal cancer: the Mercury research Project. Recent Results Cancer Res 2005; 165: 58-74.

33. Beets-Tan RG, Beets GL, Vliegen RF, Kessels AG, van Boven H, De Bruine A, et al. Accuracy of magnetic resonance imaging in prediction of tumour free resection margin in rectal cancer surgery. Lancet 2001; 357: 497-504. 